\title{
Design of A Wide Stopband Harmonic Suppressed Microstrip Low Pass Planar Filter Using Defected Ground Structure
}

\author{
Ayan Banerji \\ System Engineer \\ Infosys Ltd.
}

\begin{abstract}
In this paper an analysis is made of the filter characteristics of the popular low pass planar filter originally designed by J.S Hong and M.J Lancaster. It is noticed that a spurious harmonic at $2.9 \mathrm{GHz}$ appear in the filter characteristics. This paper presents a novel design of a wide stop band low pass planar filter using defected ground structure, which eliminates the $2.9 \mathrm{GHz}$ harmonic from the filter output. The simulation and analysis of the low pass planar filter is performed using the Ansoft HFSS v11 simulator. Snapshots of the simulation and the graphical results obtained after suppression are shown in the paper, to establish the suitability of using defected ground structure for this low pass planar filter.
\end{abstract}

Keywords: Low-pass planar filter, defected ground structure (DGS), square defect, microstrip filter.

\section{Introduction}

Wave propagation in periodic structures has been studied in applied physics for a long time [1]. Planar transmission lines with periodic structures like photonic band-gap (PBG) [2, 3, 4] have wide interest because of their pass band and stop band characteristics. But the frequencies were mostly in the optical domain. Microwave engineers adopted this idea and developed band gap structures called electronic band-gap structures (EBG) [5] by simply scaling PBG structures, producing bulky 2D and 3 D structures. Design of a steep and wide band filter required larger circuit size. The fine-tuning of the stop band was also achieved with great difficulty.

A defected structure etched in the metallic ground plane, popularly called the defected ground structure (DGS), of a microstrip filter is one of the attractive solutions to the above problem. Applications of DGS in radio frequency/microwave circuits find advantages like circuit size reduction and spurious response suppression. The conventional DGS element uses a dumbbell shaped pattern etched out in the ground plane [6]. This DGS element exhibits a band-gap characteristic at some frequency which is mainly attributed by a finite attenuation pole. The relationship between attenuation pole frequency and the physical dimensions of the DGS unit is also established. A lot of interest has developed in DGS since it has a simple equivalent circuit model and yields a low-pass property with a wide stop-band. Many research activities are reported for its application to the LPF design [7]-[12].

Nowadays, a harmonic-suppressed low-pass filter (LPF) is widely used in many communication systems to reject the spurious responses caused by power amplifiers, mixers, and oscillators. For this purpose, a lumped element such as a chip capacitor [13] or a sheet resistor [14] is incorporated in the distributed line circuits in order to break their periodicity with respect to frequency. Another approach is to employ a periodic band-gap (PBG) structure [15] or a defected ground structure (DGS). However, most of them are not focused on the suppression of harmonics, or their design procedures are so dependent upon full-wave electromagnetic (EM) optimization that it is difficult to apply the conventional LPF design method.

Received: December $16^{\text {th }}, 2010$. Accepted: Maret $9^{\text {th }}, 2012$ 
Recently an elliptic function low pass filter was developed by J.S Hong and M.J Lancaster [16] which used the Rogers RT/duroid 6010(dielectric constant=10.8) as substrate. The analysis of the filter response curve showed a second harmonic at $2.9 \mathrm{GHz}$. This paper presents a novel method to suppress this spurious second harmonic which is achieved using a conventional square dumbbell shaped defect of dimensions - length $=9.6 \mathrm{~mm}$ and slot width $=0.3 \mathrm{~mm}$.

\section{Theoritical Background}

The planar filter proposed by J.S Hong and M.J Lancaster consisted of rectangular substrate of dielectric material having dielectric constant 10.8. One side of it has a copper ground structure while the other side has a stripe of copper on it.

\section{A. Defected Ground Structure}

DGS is an etched periodic or non-periodic cascaded configuration defect in ground of a planar transmission line (e.g., microstrip , coplanar and conductor backed coplanar wave guide) which disturbs the shield current distribution in the ground plane. This disturbance changes the characteristic of a transmission line such as line capacitance and inductance. In other words, any defect etched in the ground plane of the microstrip can give rise to increasing effective capacitance and inductance. DGSs have the characteristics of stop band, slow-wave effect and high impedance. Advantages of DGS over PBG are as follows:

(1) The circuit area becomes relatively small without periodic structures. A few DGS elements can achieve similar properties like the stop-band characteristic without much periodicity.

(2) The simulated S-parameters for dumbbell DGS unit can be matched to the one-pole Butterworth-type low-pass response. In case of DGS unit, the pattern is simply fabricated and its equivalent circuit is easily extracted.

(3) DGS needs less circuit sizes for only a unit or a few periodic structures showing slowwave effect. Compared with PBG, DGS is easier to design and implement and has higher precision with regular defect structures. Therefore, it is highly convenient to extend its practical application to microwave circuits.

\section{B. Conventional DGS cell}

Figure 1(a) shows the conventional DGS cell which is composed of two ' $a \times b$ ' rectangular defected areas, and a narrow connecting slot ' $d \times w$ ' etched in backside metallic ground plane.

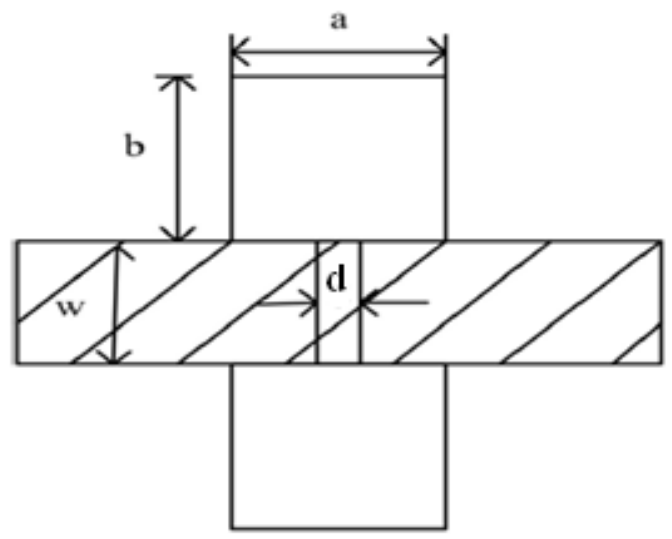

(a) 


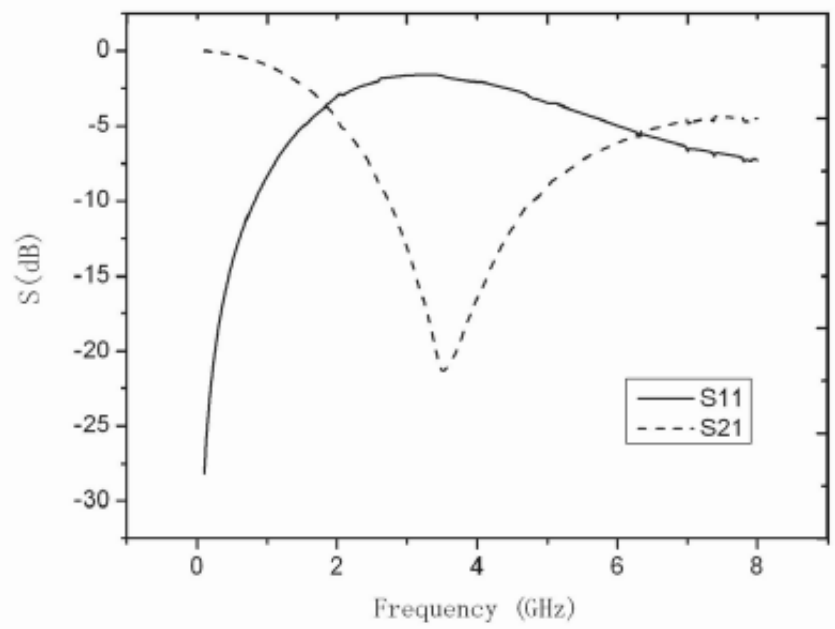

(b)

Figure 1. (a) Dumbell DGS unit (b) Simulated S-parameters for dumbell DGS unit

This is the earliest DGS used by Kim, Park et al. [5]. The S-parameters from an EM simulation of a dumbbell DGS is shown in Figure 1(b). The equivalent circuit for a DGS is a parallel-tuned circuit in series with the transmission line to which it is coupled [7]. The equivalent circuit is shown in Figure 2.

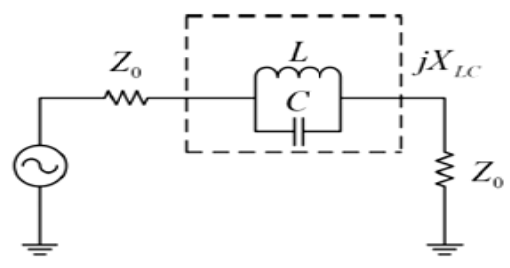

(a)

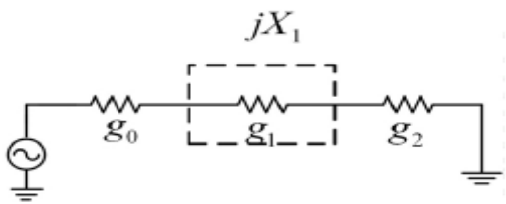

(b)

Figure 2. LC equivalent circuit : (a) equivalent circuit of the dumbell DGS circuit (b)Butterworth-type one-pole prototype low pass filter circuit.

The input and output impedances are that of the line section, while the equivalent values of L, C and R are determined by the dimensions of the DGS structure and its position relative to the transmission line as evident from the equations mentioned below.

$$
\begin{aligned}
& \mathrm{C}=\frac{\omega_{c}}{2 Z_{o}\left(\omega_{o}^{2}-\omega_{c}^{2}\right)} \\
& \mathrm{L}=1 /\left(4 \pi^{2} f_{o}^{2} C\right)
\end{aligned}
$$




$$
R(\omega)=2 Z_{O} / \sqrt{\frac{1}{\left|S_{11}(\omega)\right|^{2}}-\left(2 Z_{o}\left(\omega C-\frac{1}{\omega L}\right)\right)^{2}-1}
$$

The rectangular parts of dumbbell DGS increase route length of current and the effective inductance. The slot part accumulates charge and increases the effective capacitance of the microstrip line. Two rectangular defected areas and one connecting slot correspond to the equivalently added inductance $(L)$ and capacitance $(C)$, respectively. Accordingly, a resonance occurs at a certain frequency because of the parallel $L-C$ circuit. Inversely, it is intuitively known that the equivalent circuit, including a pair of parallel inductor-capacitor forms the resonant phenomenon in the $S$-parameter.

\section{Original Filter}

Figure 3(a) shows the original structure of the filter proposed by J.S Hong and M.J Lancaster [16]. The equivalent circuit of the filter is shown in Figure 3(b). It is a 8th ordered low pass filter with a second harmonic at $2.9 \mathrm{GHz}$ as evident from its characteristics curve shown in Figure 4.

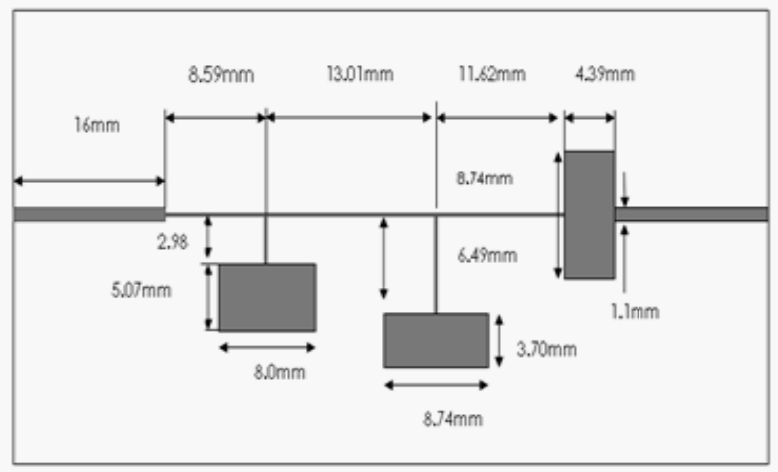

(a)

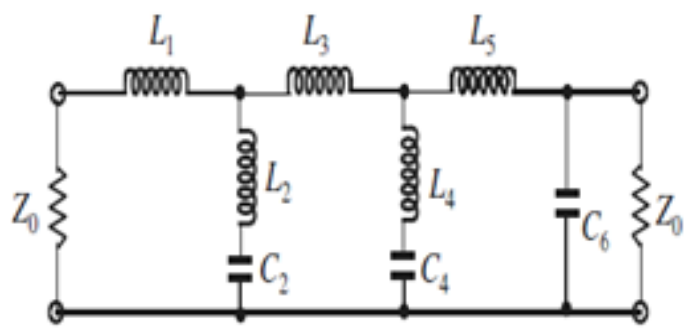

(b)

Figure 3. (a) Original Structure proposed by J.S. Hong and M.J. Lancaster

(b) Equivalent Circuit

The thickness of the substrate usually called the substrate width is $1.27 \mathrm{~mm}$, while the thickness of the copper ground usually called ground width is $0.03 \mathrm{~mm}$. The characteristics of the original filter proposed by Hong and Lancaster is shown in the Figure 4 


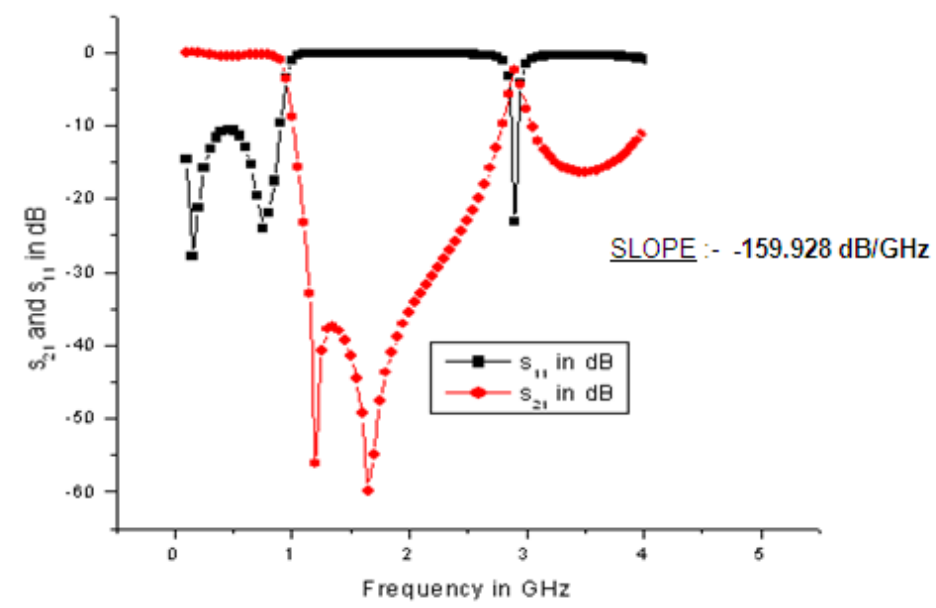

Figure 4. Characteristics of the original structure

\section{Simulation and Analysis}

In Figure 1 (a) assuming a square structure where $b=a$. The length of the square defect i.e. the value of ' $a$ ' is varied and the performance of the filter is studied. Using parametric analysis it is observed that as the etched area of the unit lattice increases, the effective series inductance increases. Further, increasing the series inductance gives rise to a lower cutoff frequency. This is evident from the simulation response curves of Figure 5, where ' $a$ ' is the length of the square defect.

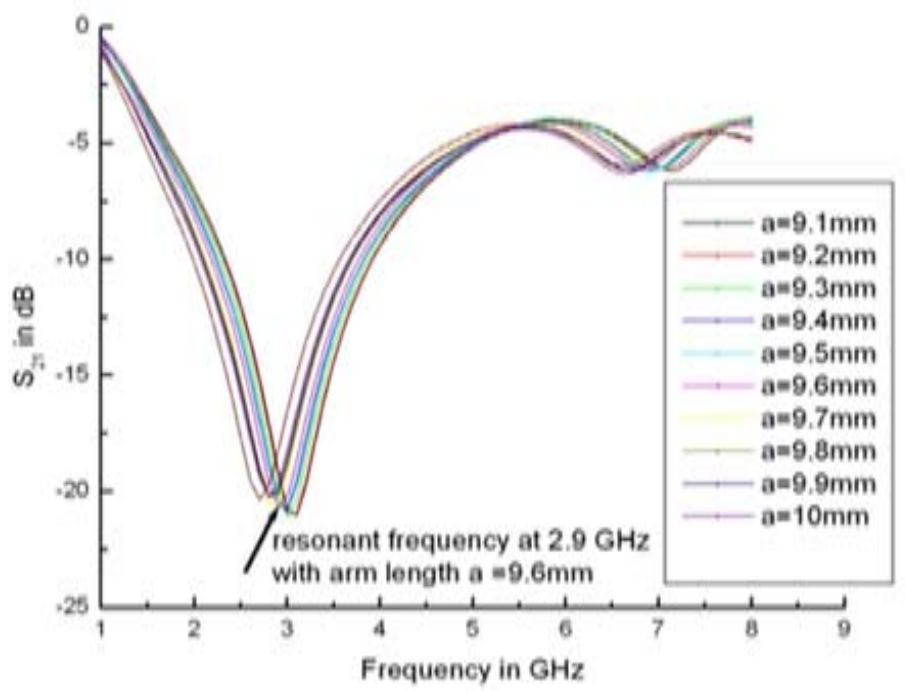

\section{a-> Length of the square defect}

Figure 5. Response curve of the parametric analysis with the variation of length of square defect.

When the etched gap distance ' $\mathrm{d}$ ' is increased, the effective capacitance decreases and this causes the attenuation pole location to move up to a higher frequency. Parametric analysis is carried out by varying the gap ' $d$ '. The result of analysis is shown in Figure 6. The movement of the attenuation pole location to higher frequency is clearly brought out in the simulation. 


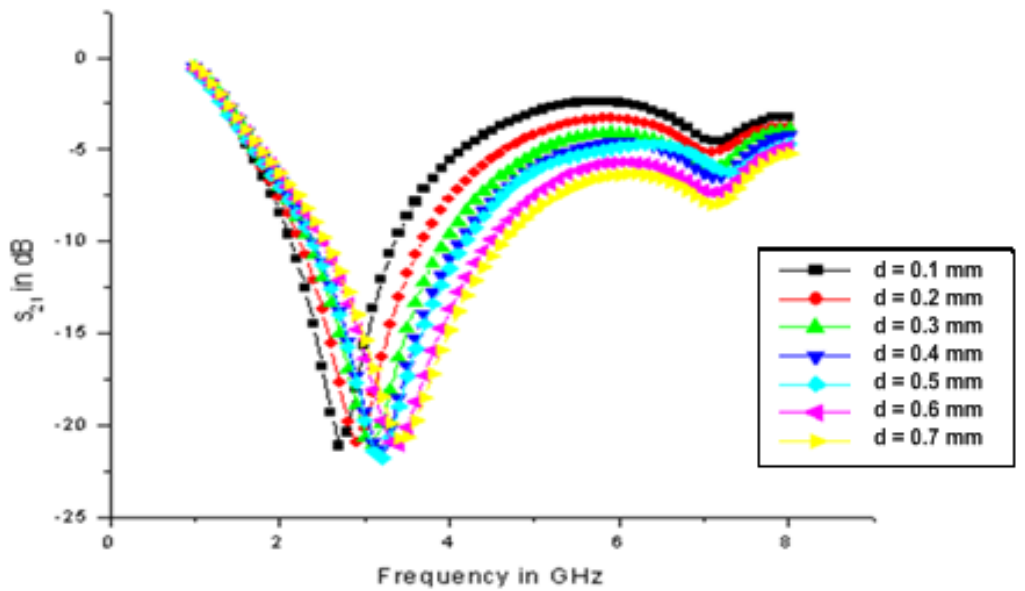

Figure 6. Response curve of the parametric analysis with the variation of slot width ' $d$ '.

Figure 7 shows the dumbbell shaped defect with appropriate dimensions. The dimensions were found using parametric analysis in order to suppress the $2.9 \mathrm{GHz}$ harmonic in the response of the original filter.

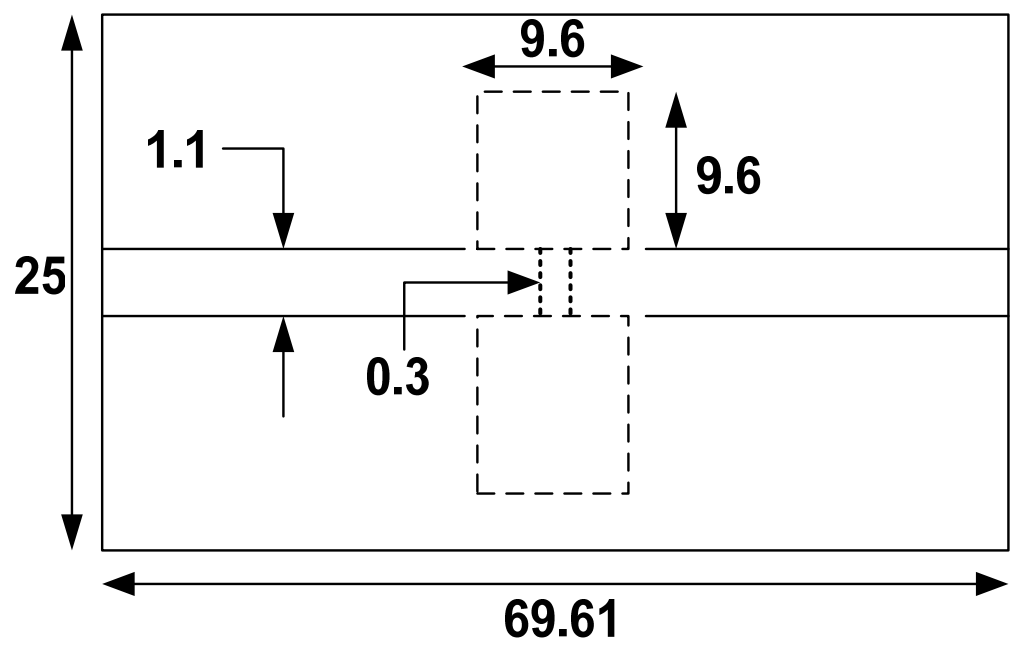

Figure 7. Square Dumbbell Shaped Defect with Appropriate Dimensions

\section{E-Field transmission of the square defect}

Figure 8 shows the snapshots of simulation at various frequencies of the $\mathrm{E}-$ Field transmission of the dumbbell shaped defect having the dimensions as shown in Figure 7. 


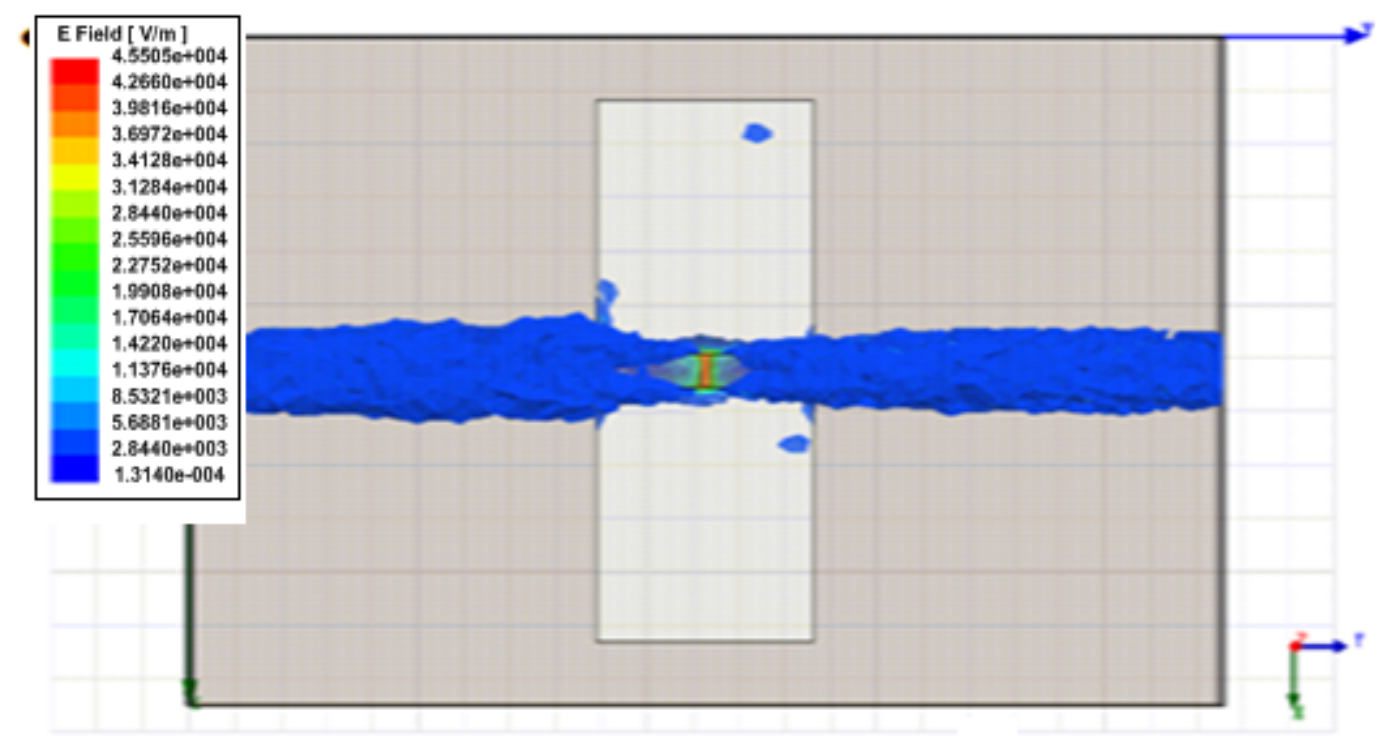

Figure 8. (a) Snapshot of E-Field transmission at $0.5 \mathrm{GHz}$

The E-Field snapshot at $0.5 \mathrm{GHz}$ shown in Figure $8(\mathrm{a})$ depicts that at $0.5 \mathrm{GHz}$ the signal is allowed to pass through as represented by the blue colour.

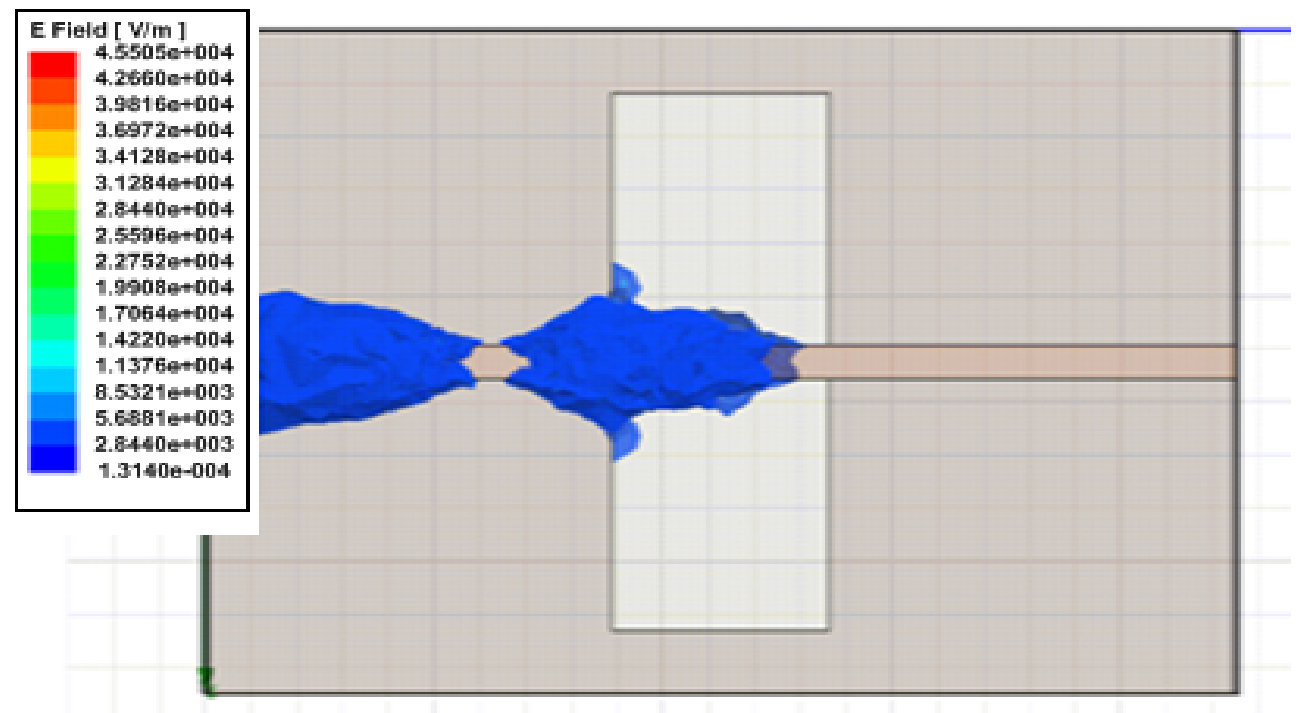

Figure 8. (b) Snapshot of E-Field transmission at $2.9 \mathrm{GHz}$.

The E-Field snapshot at $2.9 \mathrm{GHz}$ shown in Figure 8(b) depicts that at $2.9 \mathrm{GHz}$ the signal is not allowed to pass through and no output is obtained at the other end.

\section{E-Field transmission of the original filter}

Figure 9 shows the snapshots of simulation of the E-Field transmission by the original filter designed by J.S Hong and M.J Lancaster at various frequencies. 


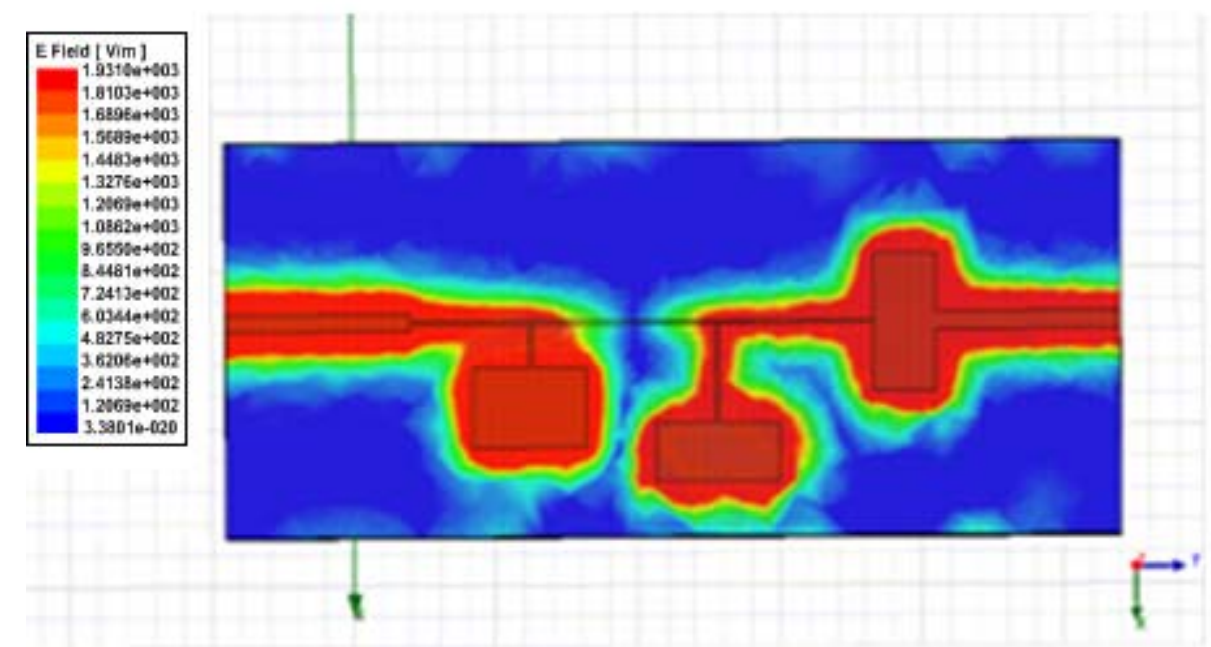

Figure 9(a) Snapshot of E-Field Trasmission at $0.5 \mathrm{GHz}$ (Passband)

The E-Field snapshot at $0.5 \mathrm{GHz}$ shown in Figure 9(a) depicts that at $0.5 \mathrm{GHz}$ the signal is allowed to pass through by the original filter.

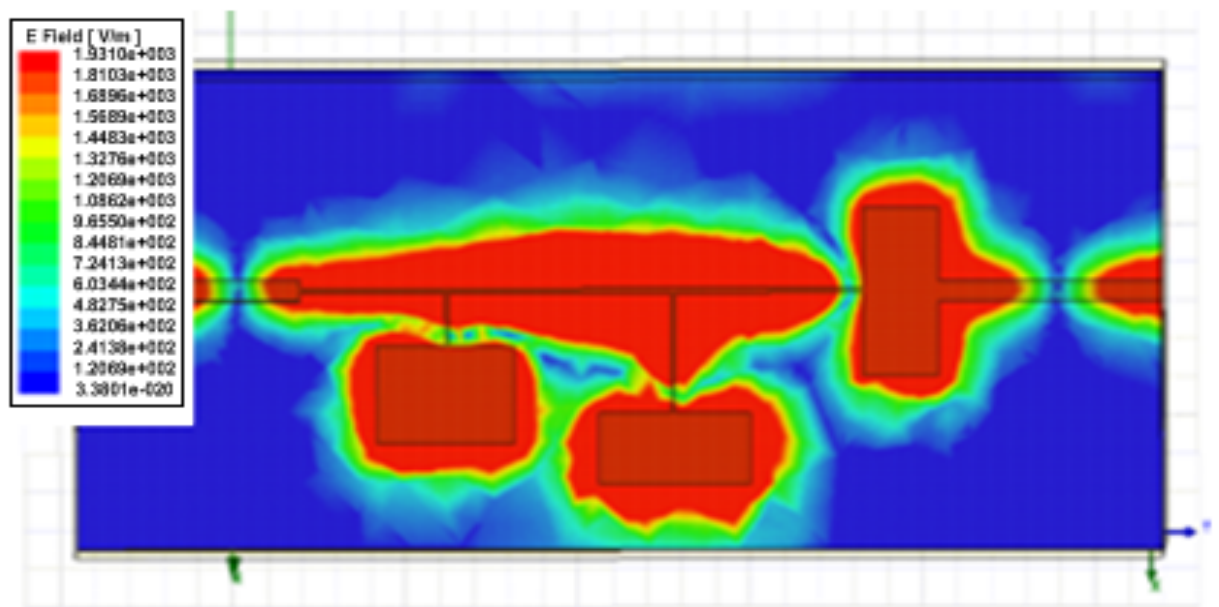

Figure 9(b) Snapshot of E-Field Trasmission at 2.9 GHz

The E-Field snapshot at $2.9 \mathrm{GHz}$ shown in Figure 9(b) depicts that at $2.9 \mathrm{GHz}$ the signal is allowed to pass through as represented by the original filter. This harmonic in the output of the original filter deteriorates its performance. The objective of the present work is to suppress the $2.9 \mathrm{GHz}$ harmonic in the response of the original filter. 


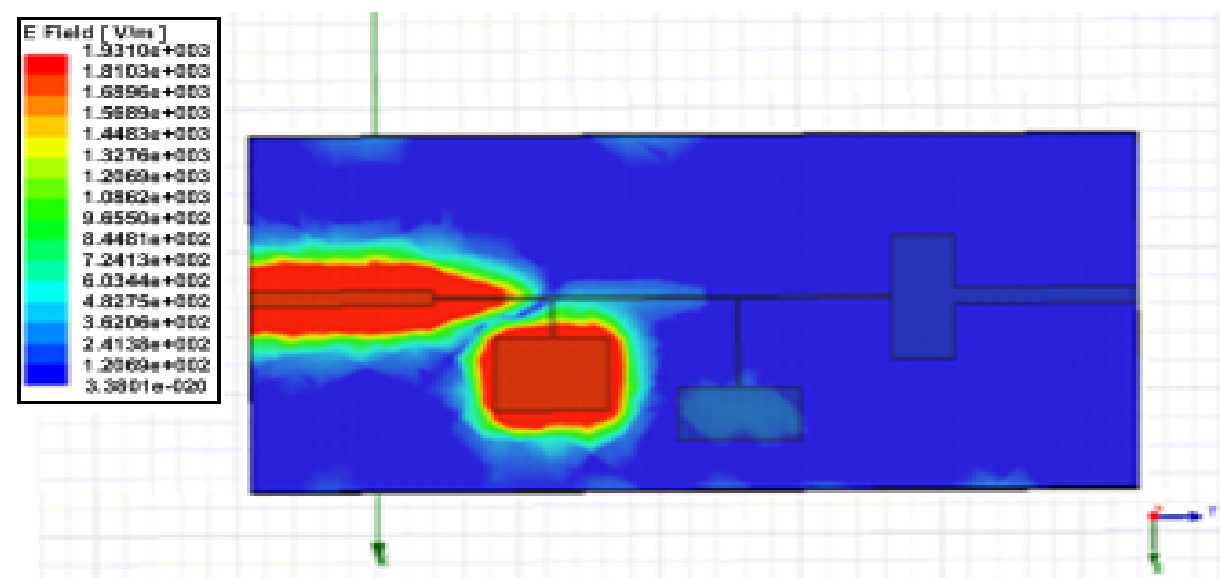

Figure 9(c) Snapshot of E-Field Trasmission at $1.5 \mathrm{GHz}$

The E-Field snapshot at $1.5 \mathrm{GHz}$ shown in Figure 9(c) depicts that at $1.5 \mathrm{GHz}$ the signal is not allowed to pass through as frequency is beyond the cutoff frequency of the original filter, which is depicted in Figure 4.

Analysis of the filter with single defect

Figure10 shows the original filter modified with a single defect etched out of the ground plane beneath the $50 \Omega$ line on other side of the substrate.

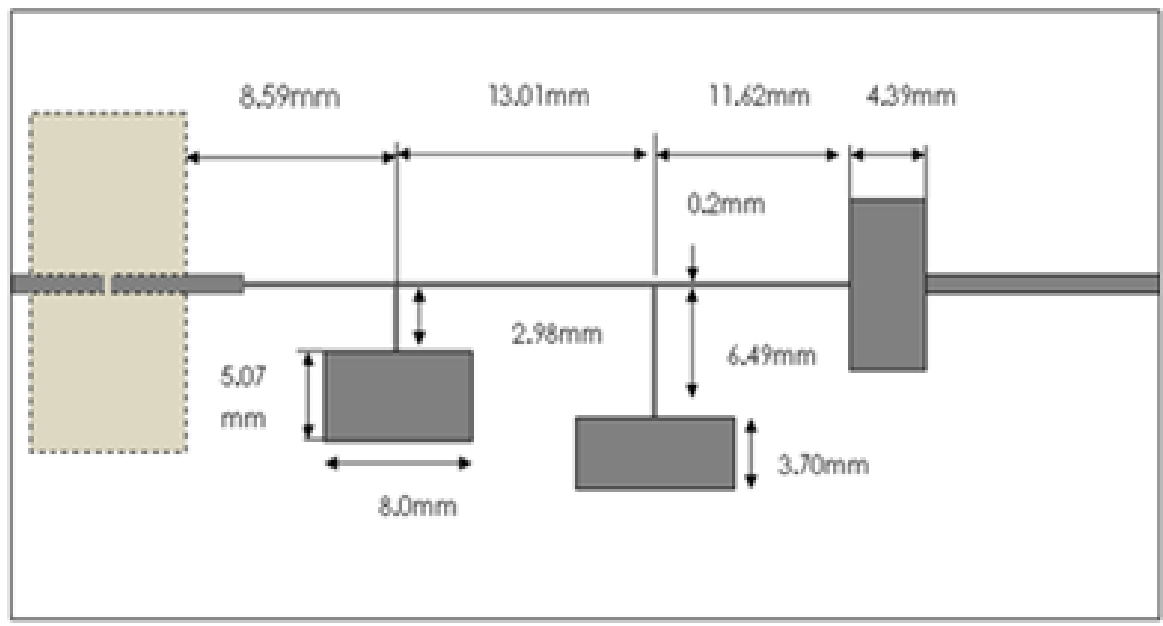

Figure 10. Filter with single defect

Simulation of the structure shows that there is a reduction of $20 \mathrm{~dB}$ in the power level at 2.9 $\mathrm{GHz}$ which is the spurious pass-band in the original filter. Comparison of the characteristic curve of the original filter with the characteristic curve of the filter structure with single defect is shown in Figure 11. 


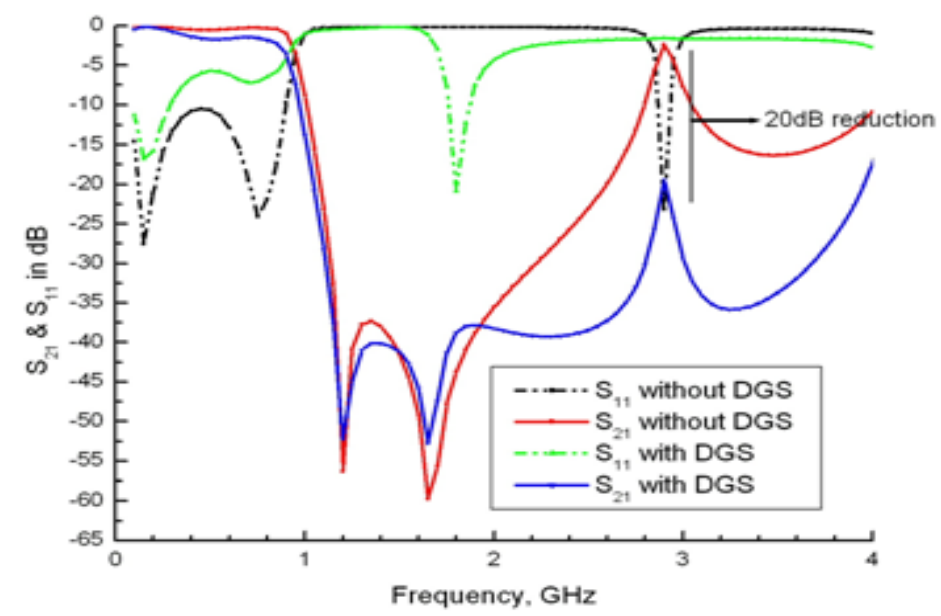

Figure 11. Comparison of the characteristic curves

\section{E-Field transmission with single defect}

Figure 12 shows the snapshots of the E-Field transmission of the original structure modified with a single defect at various frequencies during simulation. Observation shows that there is very little transmission at $2.9 \mathrm{GHz}$ when the single defect is introduced in the original structure.

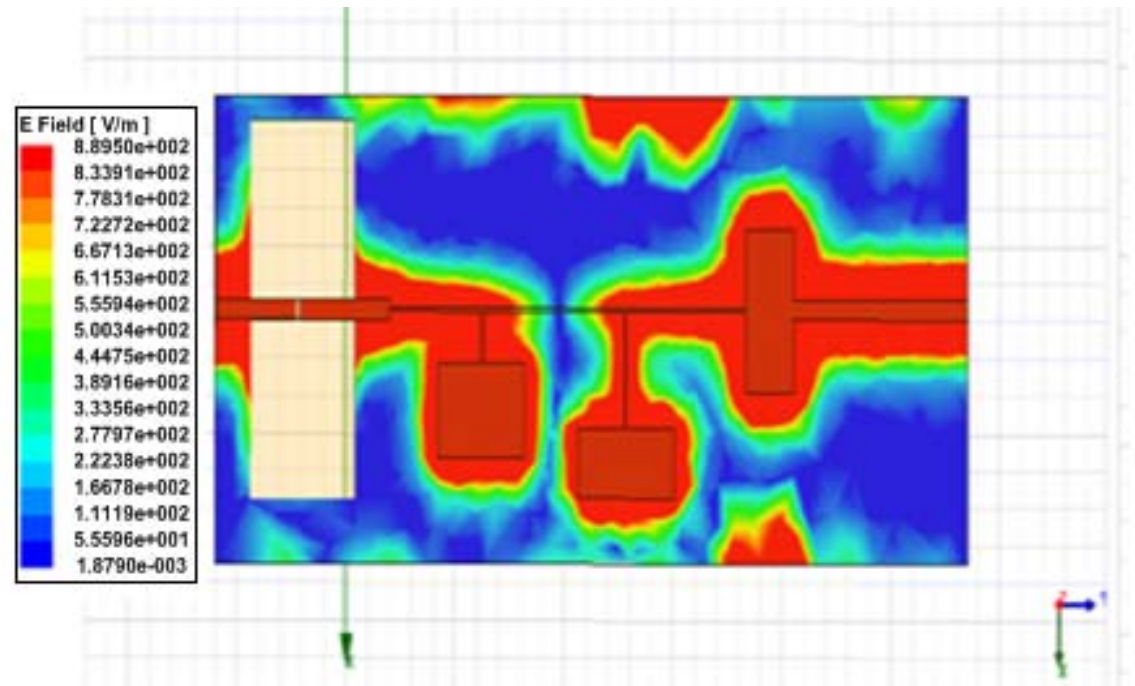

Figure 12(a) Snapshot of E-Field Trasmission at $0.5 \mathrm{GHz}$

The E-Field snapshot at $0.5 \mathrm{GHz}$ shown in Figure 12 (a) depicts that at $0.5 \mathrm{GHz}$ the signal is allowed to pass through by the modified filter. 


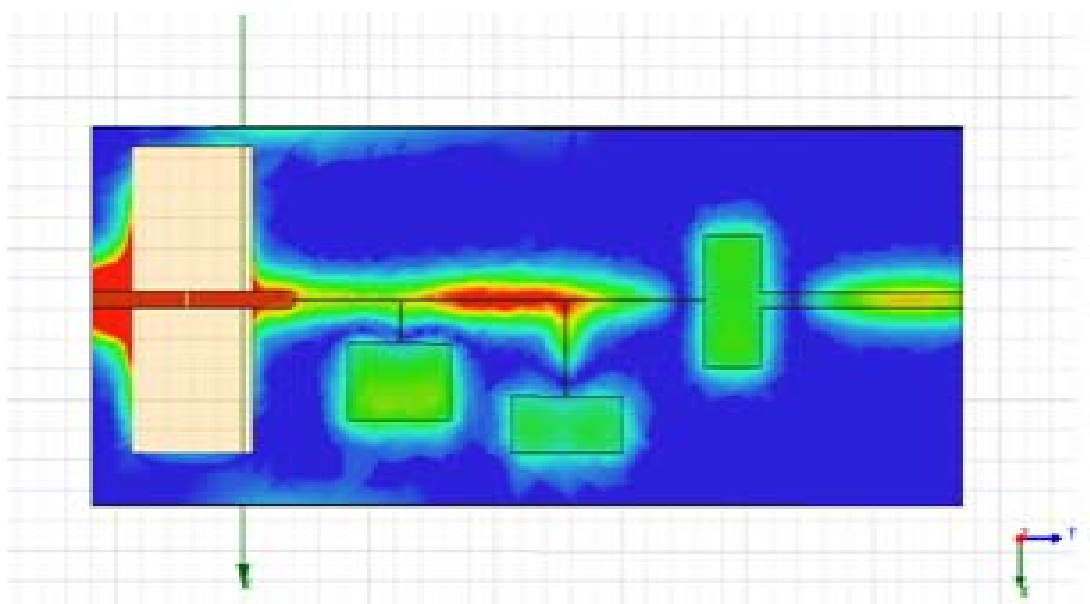

Figure12 (b) Snapshot of E-Field Trasmission at $2.9 \mathrm{GHz}$

The E-Field snapshot at $2.9 \mathrm{GHz}$ shown in Figure 12(b) depicts that at $2.9 \mathrm{GHz}$ the signal is not allowed to pass through by the modified filter. The objective of the present work to suppress the $2.9 \mathrm{GHz}$ harmonic in the response of the original filter is thus achieved.

\section{Analysis of the filter with two defects}

To analyze the effect of more defects on the response of the filter, an identical defect is introduced at the other end. Figure 13 shows the original structure modified with two defects, etched out at both ends in the ground plane beneath the $50 \Omega$ lines on the other side of the substrate.

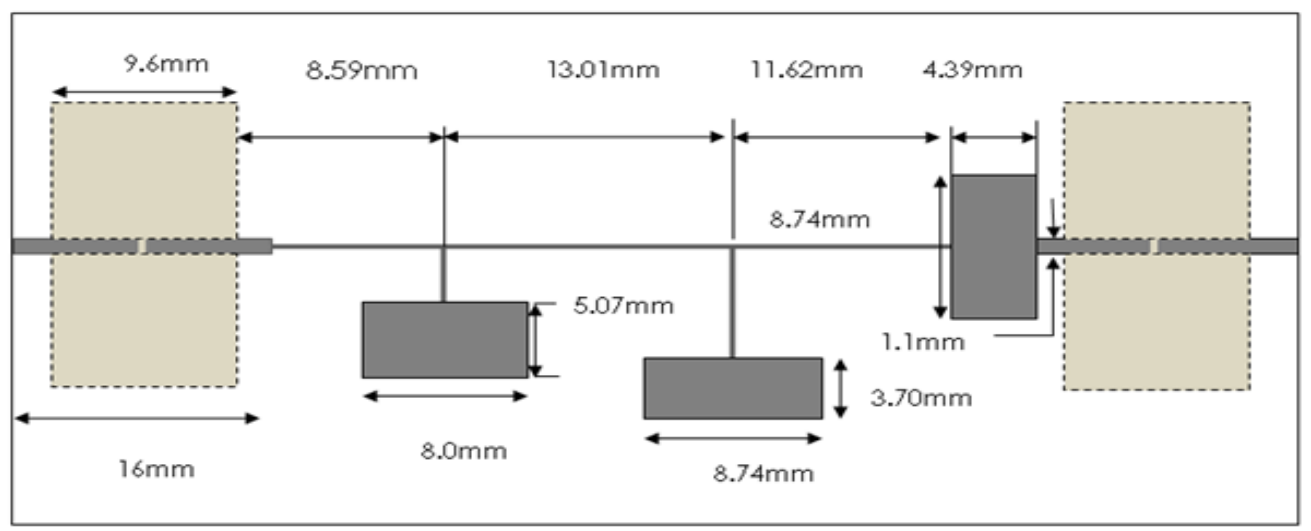

Figure 13. Filter with two defects.

Simulation of the filter with two defects shows that there is a reduction of $30 \mathrm{~dB}$ in the power level at $2.9 \mathrm{GHz}$. Comparison of the characteristic curve of the original structure with the characteristic curve of the structure with two defects is shown in Figure13. Further it is noticed that the ripple in the pass band becomes more pronounced than in the case with the single defect structure as shown in Figure 11. 


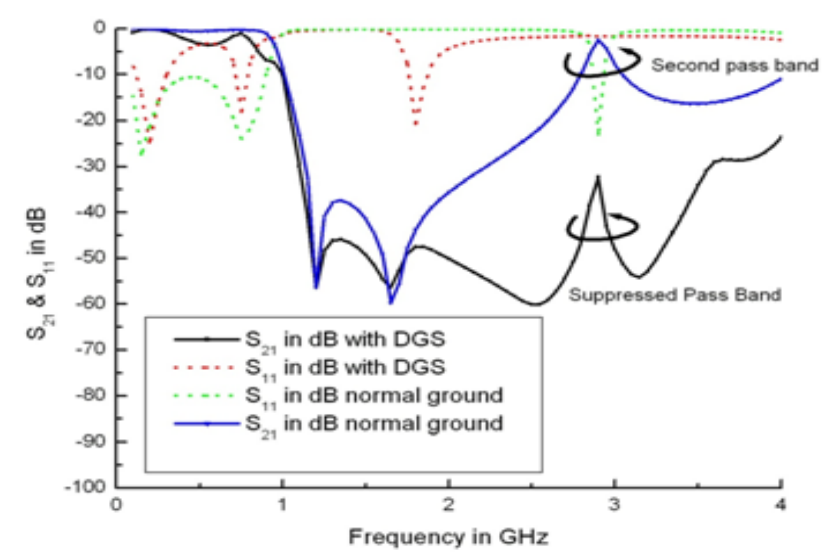

Figure 14. Comparison of characteristic curves

\section{E-Field transmission with two defects}

Figure 15 shows the snapshots of the E-Field transmission of the original structure modified with two defects at various frequencies during simulation. Observation shows that there is very little transmission at $2.9 \mathrm{GHz}$ when two defects are introduced in the original structure.

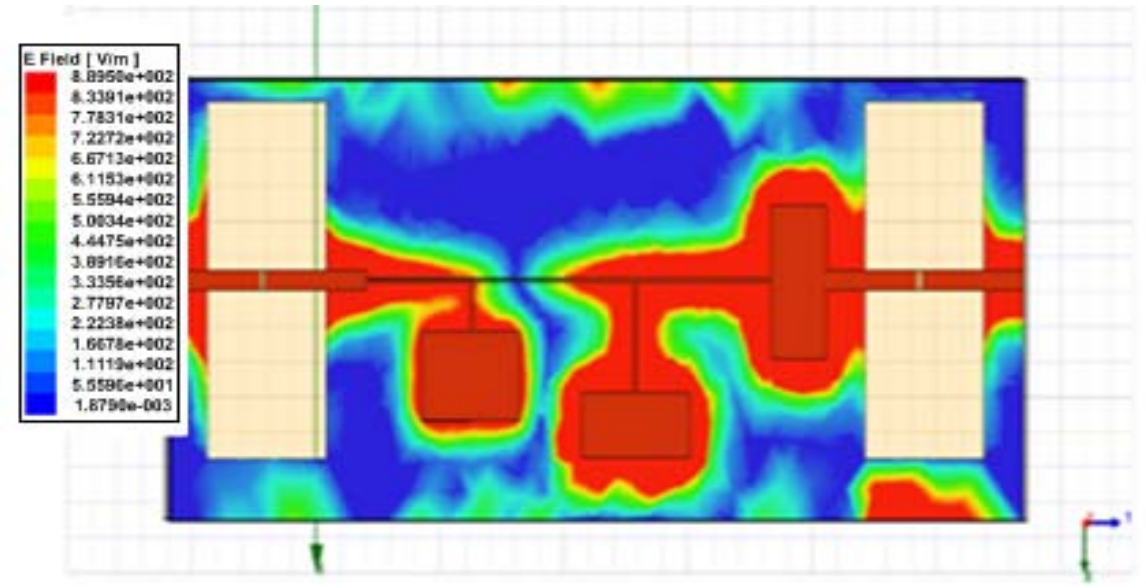

Figure 15(a) Snapshot of E-Field Trasmission at $0.5 \mathrm{GHz}$

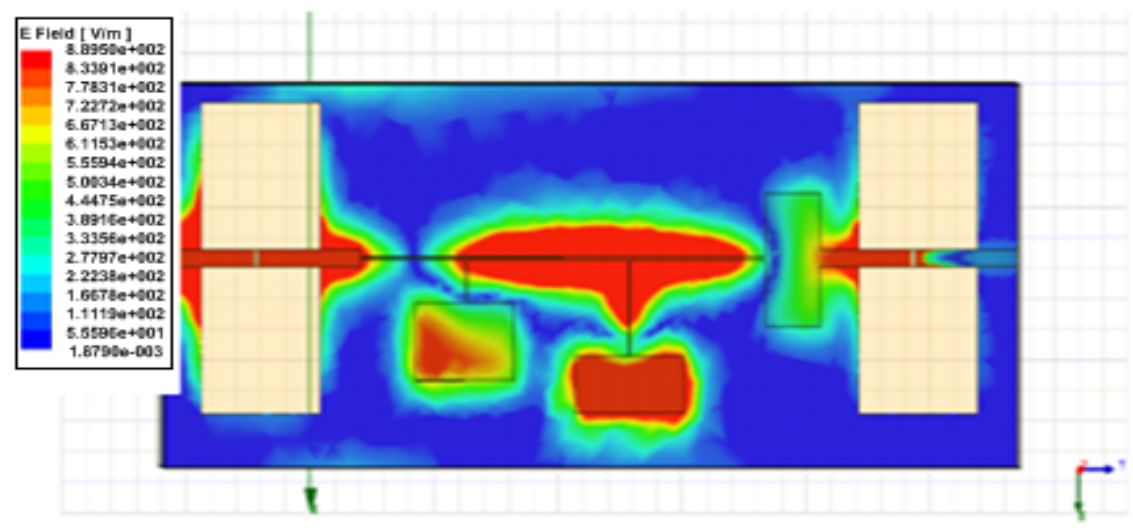

Figure 15(b) Snapshot of E-Field Trasmission at $2.9 \mathrm{GHz}$ 
The E-Field snapshot at $0.5 \mathrm{GHz}$ shown in Figure 15 (a) depicts that at $0.5 \mathrm{GHz}$ the signal is allowed to pass through by the modified filter with two defects.

The E-Field snapshot at $2.9 \mathrm{GHz}$ shown in Figure 15(b) depicts that at $2.9 \mathrm{GHz}$ the signal is not allowed to pass through by the modified filter with two defects and better attenuation of about $30 \mathrm{~dB}$ at $2.9 \mathrm{GHz}$ is obtained.

\section{Conclusion}

This paper establishes the suitability of using conventional dumbbell shaped defected ground structure for elimination of $2.9 \mathrm{GHz}$ harmonic for the low pass planar filter designed by J.S. Hong and M.J. Lancaster. A comparison has been made using a single defect and then using two defects. The simulation firmly establishes the fact that when a single defect is introduced there is reduction of $20 \mathrm{~dB}$ in the power level at $2.9 \mathrm{GHz}$, the spurious frequency in the pass band and when two defects are introduced there is a reduction of $30 \mathrm{~dB}$ in the power level at $2.9 \mathrm{GHz}$ which is better than when a single defect is introduced. However more ripples are introduced in the pass band in case of the filter structure with two defects. A $20 \mathrm{~dB}$ reduction is also a very considerable reduction in the harmonic. When a trade off is made between ripples in the pass band and reduction of the spurious pass band at $2.9 \mathrm{GHz}$, the filter with single defect shows much better performance than the filter with two defects. Thus the original low pass planar filter of Hong and Lancaster when modified with a single dumbbell shaped defected ground structure produces a much better performance. This new high performance filter is very compact and can be easily packaged in microwave components and antenna arrays.

\section{References}

[1] E.Yablonovitch, "Photonic crystals," J. Modern Opt., vol.41, no. 2, pp. 173-194, 1994.

[2] S. John, "Strong localization of photons in certain disordered Dielectric superlattices," Physical Review Letters, vol. 58, no. 23, pp. 2486-2489, 1987.

[3] V. Radisic, Y. Qian, R. Coccioli, T. Itoh, "Novel 2-D photonics bandgap structure for microstrip lines," IEEE Microwave Guided Wave Lett., vol. 8, no. 2, pp. 69 - 71, 1998.

[4] S. K. Parui, S. Das, "A simple electromagnetic bandgap structure for microstrip line," Proceedings of the IEEE India Annual Conference. pp. 547-548, 2004.

[5] C. S. Kim, J. S. Park, D. Ahn, and J. B. Lim, "A novel 1-D periodic defected Ground structure for planar circuits," IEEE Microw. Wireless Compon. Lett., vol. 10, no. 4, pp. 131-133, Apr. 2000

[6] D. Ahn, J. S. Park, C. S. Kim, J. Kim, Y. X. Qian, and T. Itoh, "A design of the lowpass filter using the novel microstrip defected ground structure," IEEE Trans. Microw. Theory Tech., vol. 49, no. 1, pp. 86-93, Jan. 2001.

[7] J. S. Park, J. H. Kim, J. H. Lee, S. H. Kim, and S. H. Myung, "A novel equivalent circuit and modeling method for defected ground structure and its application to optimization of a DGS lowpass filter," IEEE MTT-S Int. Dig., pp. 417-420, 2002.

[8] A. B. Abdel-Rahman, A. K. Verma, A. Boutejdar, and A. S. Omar, "Control of bandstop response of Hi-Lo microstrip lowpass filter using slot in ground plane," IEEE Trans. Microw. Theory Tech. vol. 52, no. 3, pp. 1008-1013, Mar. 2004.

[9] N. C. Karmakar and M. N. Mollah, "Investigation into nonuniform Photonic bandgap microstripline low Pass filters," IEEE Trans Microw. Theory Tech., vol. 51, no. 2, pp. 564-572, Feb. 2003.

[10] H. W. Liu, Z. F. Li, X. W. Sun, and J. F. Mao, "An improved 1-D periodic Defected Ground structure for microstrip line," IEEE Microw. Wireless Compon. Lett. vol.14, no. 4, pp. 180-182, Apr. 2004.

[11] R. Zhang and R. R. Mansour, "A Novel lowpass microstrip filter using Metal loaded slots in the ground plane," IEEE MTT-S. Int. Dig., pp. 1311-1314, Jun. 2004.

[12] J. W. Sheen, "A compact semi lumped low-pass filter for harmonics and spurious suppression," IEEE Microw. Guided Wave Lett., vol. 10, no. 3, pp. 92-93, Mar. 2000. 
[13] M. Q. Lee, K. K. Ryu, I. B. Yom, and S. P. Lee, "Lowpass filter for spurious suppression," Electron. Lett., vol. 38, no. 5, pp. 227-228, Feb. 2002.

[14] F.-R. Yang, Y. Qian, and T. Itoh, "A novel uniplanar compact PBG structure for filter and mixer applications," IEEE MTT-S Int Dig., vol. 3, pp. 919-922, Jun. 1999.

[15] J. I. Park, C. S. Kim, et al., "Modeling of a photonic band gap and its application for the low-pass filter design," Asia Pacific Microwave Conf. Proc. APMC, Vol. 2, pp 331- 334, 1999.

[16] J. S Hong and M.J. Lancaster "Microstrip Filters for RF/Microwave Applications," John Wiley \& Sons, Inc, New York, 2001.

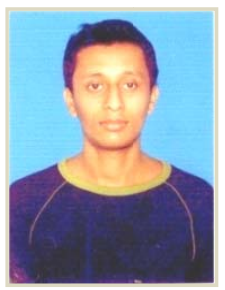

Ayan Banerji, He has graduated in Electronics and Communication Engineering from Institute of Technology \& Marine Engineering, W. Bengal, India in the year 2010. The research on Low pass planar filter using Defected Ground Structure was carried out by him during his under graduate studies. He is presently posted as System Engineer at the INFOSYS Ltd. Chennai, India. His areas of interest are Microwave engineering, Power Electronics, and Software engineering. 